\title{
Reconstruções orbitárias: relato de três casos
}

\section{Orbital reconstructions: three case reports}

\author{
Rafael Moreira Daltro* \\ Leila Guerreiro de Jesus ${ }^{* *}$ \\ Cleidiana Celi Bomfim Oliveira** \\ Lucas da Silva Barreto ${ }^{* * * *}$ \\ Paloma Heine Quintas $s^{* * * * *}$ \\ Vildeman Rodrigues de Almeida Junior ${ }^{* * * * * *}$
}

\section{Resumo}

Objetivo: este trabalho visa a apresentar uma série de três casos clínicos com diferentes condutas para tratamento de fraturas de assoalho de órbita. Relato de casos: no primeiro caso, o paciente, sexo masculino, foi vítima de ferimento por arma de fogo, apresentando diplopia, trismo e queixas estéticas. A conduta seguida foi reconstrução do assoalho da órbita com tela de titânio isolada. No segundo caso, o paciente, sexo masculino, foi vítima de acidente motociclístico, apresentando diplopia e enoftalmo. A conduta seguida foi reconstrução utilizando associação de tela de titânio com polimetilmetacrilato (PMMA). No terceiro caso, o paciente, sexo masculino, foi vítima de acidente automobilístico, apresentando enoftalmo, diplopia e perda de projeção malar. A conduta seguida foi reconstrução utilizando associação da tela de titânio com PMMA. Nos três casos, os pacientes evoluíram com melhora do quadro clínico, mostrando-se satisfeitos com os resultados estético e funcional. Considerações finais: embora as condutas sejam diversificadas e variem entre cirurgiões, é importante o conhecimento das técnicas e dos materiais a serem utilizados, bem como das vantagens e desvantagens de cada intervenção, sendo de fundamental importância também a avaliação de cada caso em particular.

Palavras-chave: Face. Fraturas ósseas. Órbita.

\section{Introdução}

As órbitas são dois espaços simétricos localizados no terço médio da face. Elas são limitadas superiormente pela calota craniana, medialmente pelas células etmoidais, inferiormente pelo seio maxilar e lateralmente pela fossa zigomática. Cada espaço orbitário é formado por sete componentes ósseos, sendo eles: face orbital do osso frontal; face orbital do osso zigomático; face orbital da maxila; osso lacrimal; lâmina orbitária do osso etmoide; face orbital do osso esfenoide; processo orbitário do osso palatino $^{1}$.

Por conta da sua projeção e do posicionamento em face, a órbita tem uma maior susceptibilidade a traumas. Sua parede medial e seu assoalho, devido à localização de espaços pneumáticos adjacentes, são mais frágeis e, por isso, mais comumente fraturados $^{2}$. A forma diagnóstica desse tipo de trauma é clínica e imaginológica. Os exames imaginológicos são muito valorizados para conclusão diagnóstica; entre eles, estão as radiografias ântero-posteriores de Waters e Caldwell, além das tomografias computadorizadas e ressonâncias magnéticas ${ }^{3}$.

As fraturas orbitárias são classificadas em: intraorbitárias (blow-in), que ocorre quando a pressão do trauma impulsiona o conteúdo do seio maxilar 
para a cavidade orbitária; ou intrassinusais (blow-out), que ocorre quando a lesão propicia pressão na órbita, de modo que seus tecidos se desloquem para o interior do seio maxilar ou das células etmoidais ${ }^{2}$. Os pacientes que apresentam fraturas de órbita costumam ter como sinais clínicos o edema e a equimose periorbitários, assimetria das órbitas, limitação da motilidade ocular, ptose palpebral, enoftalmo e hipoftalmo. É recorrente, também, que os pacientes apresentem sintomas como parestesia na região infraorbitária e diplopia ${ }^{4}$.

A forma de tratamento das fraturas de assoalho não é consenso na literatura. Alguns autores sustentam que se deve aguardar até quatorze dias após o trauma para avaliar se os sintomas, como diplopia e enoftalmo, sessam com a redução do edema, uma vez que essas queixas podem reduzir espontaneamente, sem necessidade de intervenção cirúrgica ${ }^{5}$. Outros autores ${ }^{6}$ defendem que esse período de espera deve ser entre quatro e seis meses. Enquanto outros afirmam que as fraturas sintomáticas devem receber tratamento cirúrgico imediato para minimizar sequelas ${ }^{7}$. Em relação aos casos de intervenção cirúrgica, estudos apontam que melhores resultados são obtidos se executada a intervenção até quatorze dias após o trauma ${ }^{1}$.

Assim como a forma de tratamento, o material utilizado para reconstrução orbitária também é objeto de debate entre autores ${ }^{8}$. Existe uma discussão entre preferências por materiais de enxertos, materiais aloplásticos ou a utilização de ambos. Em relação aos enxertos, eles podem ser ósseos ou cartilaginosos, sendo considerados padrão-ouro; entretanto, apresentam como desvantagens maior morbidade e tempo cirúrgico. Os aloplásticos são, principalmente, as telas de titânio e polimetilmetacrilato (PMMA), podendo ser utilizadas isoladas ou em associação; esses materiais apresentam como vantagem redução de custos e boa estabilidade .

Com o desenvolvimento de novas tecnologias, houve ganhos em exames, mensurações e teste de materiais em protótipos individuais, sendo possível atualmente melhor planejamento, tornando o ato cirúrgico mais rápido, e os resultados obtidos, melhores. Estudos observam que o uso de planejamento virtual com prototipagem 3D é uma boa ferramenta para garantir bom posicionamento de implantes aloplásticos e reanatomização orbitária ${ }^{10}$.

A finalidade deste estudo foi relatar uma série de três casos clínicos com diferentes condutas para tratamento de fraturas de assoalho de órbita, proporcionando uma discussão sobre o tema.

\section{Relato de casos clínicos}

Todos os pacientes participantes assinaram o termo de consentimento livre e esclarecido, autorizando o uso e a divulgação de suas informações e imagens para fins científicos.

\section{Caso 1}

O paciente buscou atendimento no ambulatório de Cirurgia e Traumatologia Bucomaxilofacial da Universidade Federal da Bahia/Obras Sociais Irmã Dulce (Ufba/Osid) quatro meses depois de ter sido vítima de ferimento por arma de fogo. Ele havia sofrido ferimentos em outras partes do corpo além da face, por isso teve atendimento odontológico apenas após a liberação das especialidades médicas. Quando compareceu ao atendimento, apresentava queixas de diplopia, de trismo e estética.

Para o tratamento, foram propostas a intervenção cirúrgica para remoção dos artefatos e a instalação de tela de titânio para reposicionamento e sustentação do globo ocular. Após a conduta adotada, pôde-se observar melhora da simetria orbitária por meio da comparação dos exames de imagem pré e pós-operatórios (Figura 1). O paciente evoluiu com melhora do quadro funcional e ficou satisfeito com o resultado estético. 

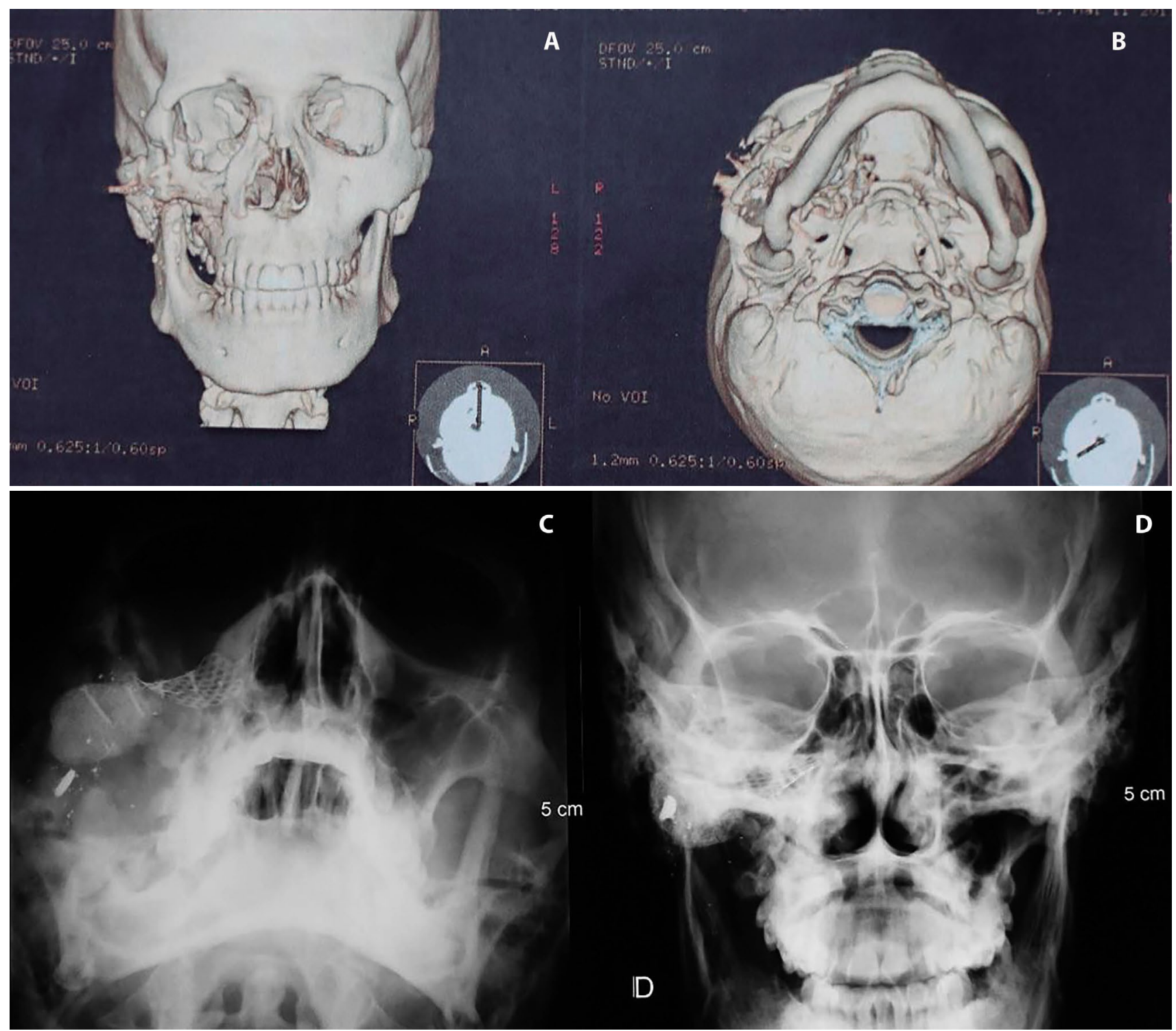

Figura 1 - Exame de imagens do caso clínico 1

Legenda: A: reconstrução 3D da tomografia computadorizada da condição pré-operatória em visão frontal; B: reconstrução 3D da tomografia computadorizada da condição pré-operatória em visão caudocraniana; C: incidência radiográfica de Waters da condição pós-operatória; D: incidência radiográfica frontal da condição pós-operatória.

Fonte: autores.

\section{Caso 2}

O paciente buscou atendimento no ambulatório seis meses após ter sido vítima de acidente motociclístico. Ele apresentava queixas de diplopia e enoftalmo. Pela imagem tomográfica, percebeu-se assimetria de volume orbitário.
A conduta, nesse caso, foi a realização um acesso subtarsal e coronal, para reposicionamento do globo e fixação com placas de titânio do rebordo orbitário inferior, do pilar frontozigomático e do arco zigomático, sendo realizada a reconstrução do assoalho de órbita com PMMA e tela de titânio (Figura 2). O paciente evoluiu, no pós-operatório, com melhoras estética e funcional. 

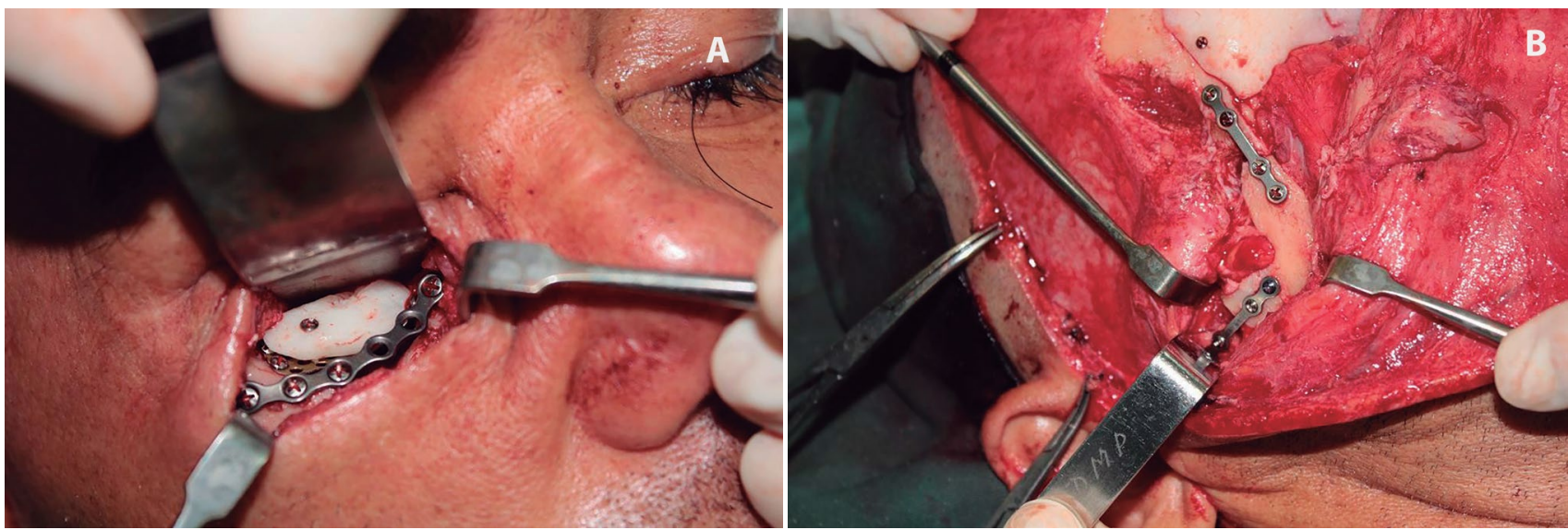

Figura 2 - Imagens transoperatórias do caso clínico 2

Legenda: A: acesso subtarsal, evidenciando fixação do enxerto de PMMA, tela de titânio e reconstrução do rebordo orbitário inferior; B: acesso coronal, mostrando a fixação dos pilares frontozigomático e do arco zigomático.

Fonte: autores.

\section{Caso 3}

O paciente compareceu no ambulatório três meses após trauma em acidente automobilístico com queixas de enoftalmo, diplopia e com perda de projeção malar.

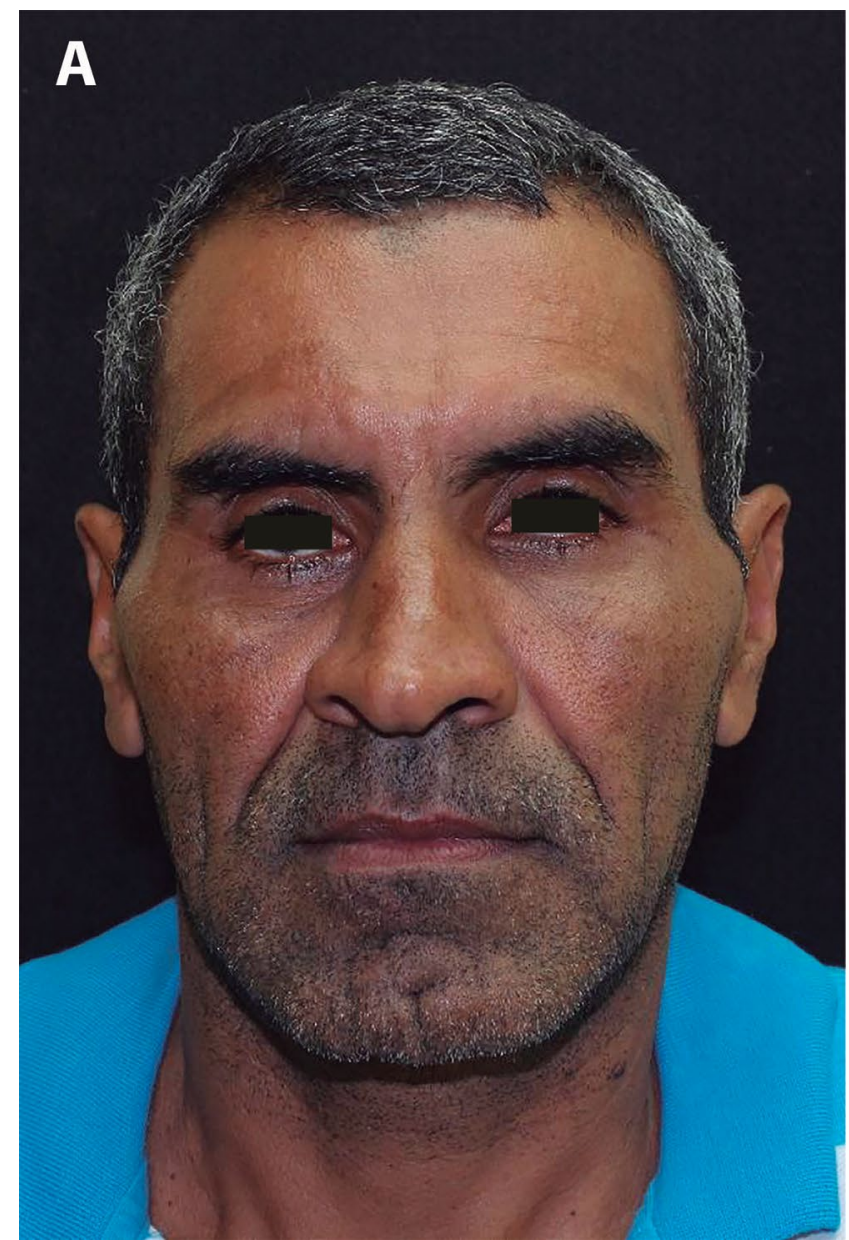

Figura 3 - Condições pré e pós-operatórias do caso clínico 3

Legenda: A: imagem frontal da condição pré-operatória do paciente com enoftalmo e perda de projeção malar do lado direito; B: imagem frontal da condição pós-operatória do paciente com melhoras estética e funcional.

Fonte: autores.
A conduta proposta foi realização de acessos subtarsal e intraoral, visando ao reposicionamento do globo ocular com reconstrução de assoalho com tela de titânio e enxerto de PMMA, além de reposicionamento e fixação do zigoma. O paciente relatou satisfação com o resultado estético e melhora do quadro funcional (Figura 3).

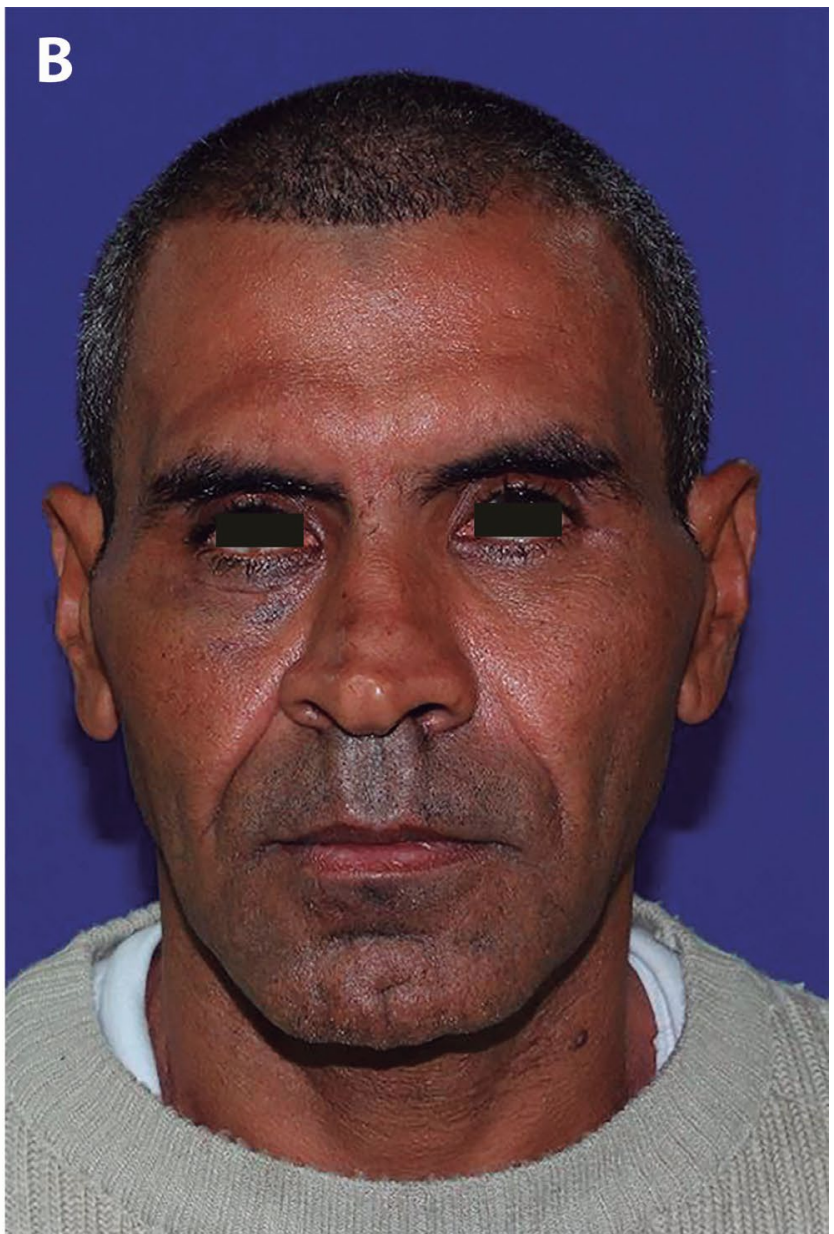




\section{Discussão}

Oliveira et al. ${ }^{11}(2012)$ afirmam em seu estudo que fraturas do tipo blow-out puras, algumas vezes, podem apresentar-se assintomáticas nos primeiros dias pós-trauma, por conta do edema de tecido mole na região, sendo necessários exames imaginológicos para correto diagnóstico. Ainda neste estudo, os autores reforçam que, pela sobreposição de estruturas, as imagens radiográficas não são eficientes para diagnóstico, sendo importante a utilização de outros recursos, como a tomografia computadorizada. Para realizar o diagnóstico das fraturas dos casos presentes neste relato, foram solicitadas tomografias computadorizadas, possibilitando ter um acurado diagnóstico pré-operatório; já, para acompanhamento do pós-cirúrgico, optou-se por radiografias de face, visando a observar o posicionamento do material de síntese óssea e a minimizar a exposição do paciente à radiação.

Su et al. ${ }^{12}$ (2015) observaram que a diplopia, sequela mais importante de fraturas de assoalho de órbita, tem padrões semelhantes em pacientes pediátricos, sendo estes pacientes os que apresentam maior duração sintomática e evolução mais lenta. Dessa forma, segundo os autores, esses pacientes devem receber tratamento cirúrgico mais imediato e associá-lo à fisioterapia para fortalecimento e recuperação da motilidade ocular. Sequelas semelhantes a esse estudo foram observadas nos casos relatados, sendo que os três pacientes apresentavam diplopia após fratura do assoalho orbitário.

A respeito da intervenção cirúrgica ou não em casos de fraturas de assoalho de órbita, Alinasab et al. ${ }^{7}$ (2014) observaram uma considerável diferença na opinião a respeito da conduta a ser seguida em situações com esse tipo de trauma. Os autores utilizaram em seu estudo exames de onze pacientes e questionaram 46 cirurgiões, com diferentes origens e experiências nesse tipo de caso, sobre a conduta mais adequada. Encontraram consenso em apenas cinco casos sobre a necessidade de cirurgia para correção sintomatológica; nos demais casos, houve discordância sobre a conduta a ser adotada. Nos casos relatados no presente estudo, todas as intervenções cirúrgicas foram realizadas depois de transcorrido um período de três meses do trauma; tal conduta foi adotada devido ao fato de aguardar a redução do edema para avaliar com maior acurácia as perdas funcional e estética.

Kashyap et al. ${ }^{13}$ (2016) aplicaram um questionário a cirurgiões, a fim de avaliar a conduta destes em casos dessas fraturas. Eles observaram que $81 \%$ dos cirurgiões são adeptos a aguardar a redução do edema para decidir pelo tratamento cirúrgico ou não. Quanto ao tempo que se deve aguardar para realizar a cirurgia, os resultados foram: $36 \%$ acham que se deve aguardar de 1 a 5 dias; $45 \%$, de 6 a 10 dias; $9 \%$, de 11 a 15 dias; e $9 \%$, acima de 15 dias.
Outro estudo ${ }^{5}$ afirmou que a intervenção cirúrgica realizada nas primeiras 24 horas pós-trauma pode estar associada a resultados imprevistos. Nos relatos desse artigo, o tempo para realização do procedimento cirúrgico foi de no mínimo três meses, assim foi possível analisar de forma mais crítica as sequelas, visto que o edema já não estava presente, facilitando o acesso cirúrgico e permitindo melhor visibilidade das referências anatômicas.

Kasaee et al. ${ }^{14}(2017)$ avaliaram a presença e a redução de sequelas como enoftalmo e diplopia em pacientes com fratura de assoalho, separando 60 pacientes em grupo cirúrgico e 72 pacientes em grupo não cirúrgico. Os autores observaram que no grupo não cirúrgico a diplopia dos pacientes diminuiu de forma considerável após três meses do trauma; já, no grupo cirúrgico, essa redução ocorreu de forma mais significativa após seis meses. Em conclusão, os autores afirmam que essa diferença se justificou pelo tempo que o paciente foi operado após o trauma, sendo ideal até 4,5 dias, e, também, pela presença de associação com a parede medial da órbita. No presente estudo, o tempo para a realização das reconstruções orbitárias foi suficiente para aguardar a redução do edema e observar melhora da sintomatologia; como não houve melhora dos sintomas, o procedimento cirúrgico foi realizado. Observamos que os três pacientes obtiveram resultados positivos, reconhecendo melhora completa da diplopia no retorno pós-operatório, geralmente um mês depois da intervenção.

Em relação à intervenção cirúrgica tardia, Scawn et $a .^{6}(2015)$ realizaram um estudo com intervenção cirúrgica após uma média de 19 meses do trauma, em que observaram que, em relação à diplopia no pré e no pós-operatórios: nenhum paciente que não apresentava o sintoma desenvolveu-o após a cirurgia; $50 \%$ dos pacientes obtiveram resolução completa; cerca de $33,3 \%$ obtiveram melhora do quadro; e cerca de $16,6 \%$ não apresentaram redução da sintomatologia. Concluíram, então, que a realização da cirurgia após seis semanas ou mais pode reduzir as sequelas, sem induzi-las, além de apresentar melhora acentuada nos aspectos estético e funcional. Resultados semelhantes foram observados nos casos expostos neste trabalho, tendo os pacientes referido resolução completa da diplopia após reconstruções orbitárias.

Nos casos em que a cirurgia é indicada, alguns materiais podem ser utilizados para reconstrução da órbita, variando entre enxertos e materiais aloplásticos. Kronig et al. ${ }^{4}(2016)$ realizaram um estudo com a reconstrução feita com enxerto ósseo autógeno e observaram que, com seguimento de um ano, nenhum dos pacientes submetidos ao procedimento apresentou enoftalmo, quando todas as paredes envolvidas foram reconstruídas; já, nos pacientes que não tiveram a parede medial reconstruída, $29 \%$ ainda apresentavam enoftalmo após o período de tempo. 
Em seu estudo, Tak et al. ${ }^{15}$ (2014) utilizaram membrana reabsorvível e matriz óssea desmineralizada para reconstrução da órbita em pacientes que apresentavam herniação de tecido ocular para o interior do seio maxilar. Eles observaram restauração da anatomia orbital em tomografia computadorizada imediatamente após o procedimento e, depois de dois anos de acompanhamento, formação óssea sem herniação tardia.

Em relação ao uso de malhas de titânio, Lee et al. ${ }^{16}$ (2017) relataram a possibilidade de síndrome de aderência orbitária. Esse fator é uma desvantagem ao uso da malha de titânio, uma vez que ocorre a formação de tecido fibrótico que pode dificultar a motilidade ocular, necessitando de uma nova cirurgia para correção dessa sequela. Sukegawa et al. ${ }^{2}$ (2017) mostraram, em contrapartida, no seu estudo, a recuperação completa da anatomia e da função orbitária por meio de reconstruções com uso desse material, desde que bem posicionado. Nos casos relatados neste trabalho com uso de tela de titânio, não foram observadas aderências, mas melhora nos aspectos estético e funcional para os pacientes.

Comparando materiais aloplásticos isolados e combinados, He et al..$^{17}$ (2012) encontraram boa projeção ocular após reconstrução orbitária em: 74\% dos pacientes tratados apenas com tela de titânio; $83 \%$, com combinação da tela de titânio e de polietileno poroso ou hidroxiapatita; $67 \%$, apenas com polietileno poroso; e $20 \%$, apenas com hidroxiapatita. Concluíram que, para tratamento de enoftalmo severo, a melhor escolha para reconstrução é a associação da tela de titânio com polietileno poroso.

Resultados semelhantes a essa associação foram observados por Han et al. ${ }^{18}$ (2016), que descreveram no seu estudo que, dos 19 casos de enoftalmo, 15 obtiveram resolução completa, e o restante obteve melhora com significância estatística, quando comparados o pré e o pós-operatórios. Consideraram, então, a associação da tela de titânio com o polietileno poroso um excelente material para reconstrução orbitária. Entretanto, Peng et al. ${ }^{19}(2017)$ não observaram diferença significativa entre essa associação e a malha de titânio isolada. Com relação à projeção ocular, nos casos relatados neste estudo, não foram observadas diferenças significativas no uso da tela de titânio e de PMMA isolados ou em associação.

A implantação da tomografia computadorizada no planejamento e a comparação das condições pré e pós-operatórias se mostram muito úteis. Sukegawa et al. ${ }^{2}$ (2017) revelam uma possibilidade de mensuração do volume orbitário anatômico por meio desse recurso. Wi et al. ${ }^{1}$ (2017), utilizando esse método, observaram que a redução do volume orbitário foi significativa imediatamente após a cirurgia e depois de seis meses. Zavattero et al..$^{20}(2017)$ encontraram volume de órbita próximo ao contralateral após a reconstrução, além de maior projeção do globo ocular, quando comparados com à condição pré-operatória. Nos casos deste estudo, não foram realizadas mensurações para verificar o volume orbitário nos momentos pré e pós-operatórios, visto que não foram realizados exames semelhantes (tomografia de face) nos dois momentos, a fim de minimizar a exposição excessiva dos pacientes à radiação.

Segundo Herford et al. ${ }^{10}$ (2017), a aquisição de técnicas de planejamento virtual e a confecção de modelos customizados, baseados na impressão $3 \mathrm{D}$, garantem precisão e acurácia no posicionamento do implante e na preservação do complexo neurovascular. Zavattero et al..$^{20}(2017)$, sobre isso, afirmaram que o uso dessa técnica obtém ganhos mais significativos, quando comparado à técnica convencional.

\section{Considerações finais}

Ainda não há consenso na literatura a respeito da conduta frente à fratura blow-out, dependendo o tratamento da escolha de cada cirurgião, bem como da particularidade de cada caso. O manejo das fraturas orbitárias assim como os dos demais traumas devem ser de conhecimento do cirurgião bucomaxilofacial, sendo importante que este domine as opções de condutas possíveis para resolução de tais casos. Diante disso, torna-se crucial o domínio dos materiais que podem ser utilizados para reconstrução da órbita, quando preciso for, e o conhecimento das suas vantagens e desvantagens.

\section{Abstract}

Objective: this study aims to present a series of three clinical cases with different approaches to the treatment of orbital floor fracture. Case reports: in the first case, the male patient was a victim of gunshot wounds, presenting diplopia, trismus, and aesthetic complaints. The treatment selected was the reconstruction of the orbital floor with an isolated titanium mesh. In the second case, the male patient was a victim of a motorcycle accident, evolving with diplopia and enophthalmos. The procedure followed was reconstruction using the association of a titanium mesh with polymethyl methacrylate (PMMA). In the third case, the male patient was the victim of an automobile accident, presenting enophthalmos, diplopia, and loss of malar projection. In this case, the reconstruction procedure was adopted using the association of a titanium mesh with PMMA. In all three cases, the patients evolved with improvement of the clinical condition, showing satisfaction with the aesthetic and functional results. Final considerations: although there are several procedures that vary among surgeons, it is important to understand the techniques and materials used, as well as the advantages and disadvantages of each intervention; it is also essential to assess each case in particular.

Keywords: Bone fractures. Face. Orbit. 


\section{Referências}

1. Wi JM, Sung KH, Chi M. 'Orbital volume restoration rate after orbital fracture'; a CT-based orbital volume measurement for evaluation of orbital wall reconstructive effect. Eye 2017; 31(5):713-9.

2. Sukegawa S, Kanno T, Shibata A, Matsumoto K, Sukegawa-Takahashi Y, Sakaida K, et al. Treatment of Orbital Fractures with Orbital-Wall Defects using Anatomically Preformed Orbital Wall Reconstruction Plate System. J Hard Tissue Biol 2017; 26(2):231-6.

3. Mororó ABG, de Almeida S, Carvalho FSR., Freire Filho FW, Bezerra M F, Tavares RN. Tratamento cirúrgico de fratura orbitária blow-out pura com tela de titânio: relato de caso clínico. Rev Odontola do Brasil Central 2014; 22(63):120-3.

4. Kronig SAJ, Van Der Mooren RJG, Strabbing M, Stam LHM, Tan JAS L, De Jongh E, et al. Pure orbital blowout fractures reconstructed with autogenous bone grafts: functional and aesthetic outcomes. Int Oral Maxillofac Surg 2016; 45(4):507-12.

5. Summers SM, Wood RM, Costello JE, Carlson CL. Orbital Trapdoor Fracture: An Open and shut Case? Western J of Emergency Med 2017; 1(1):67-8.

6. Scawn RL, Lim LH, Whipple KM, Dolmetsch A, Priel A, Korn B, et al. Outcomes of orbital blow-out fracture repair performed beyond 6 weeks after injury. Ophthalmic Plast Reconstr Surg 2015; 32(4):296-301.

7. Alinasab B, Ryott M, Stjärne P. Still no reliable consensus in management of blow-out fracture. Injury 2014; 45(1):197-202.

8. Meier PJ, Jadhav C, Morrison DA, Hayes AJ, Cunneen S. A novel individual reconstruction of a medial orbital wall blow-out fracture using a bone graft molded intraoperatively using a 3-D model: a case report. Oral Maxillofac Surg Cases $2016 ; 2(2): 19-21$

9. Mahipathy SRRV, Manavazhagan D, Durairaj AR, Sundaramurthy N, Jesudasan J S. Titanium mesh in orbital wall reconstruction: a short review. An of Applied Bio-Sciences 2017; 4(1):R1-3.

10. Herford AS, Miller M, Lauritano F, Cervino G, Signorino F, Maiorana $\mathrm{C}$. The use of virtual surgical planning and navigation in the treatment of orbital trauma. Chin J Traumatol 2017; 20(1):9-13.

11. Oliveira MTF, Zoccoli LVJ, Rodrigues ÁR, Furtado LM, Barbosa DZ. Fratura orbitária tipo blow-out pura em criança: relato de caso clínico. Rev Odontol UNESP (Online) 2012; 41(2):139-42.

12. Su Y, Shen Q, Lin M, Fan X. Diplopia of pediatric orbital blowout fractures: a retrospective study of 83 patients classified by age groups. Medicine 2015; 94(4):1-6.

13. Kashyap R, Mittal G, Kataria G. Orbital blowout fractures: A Survey and review. Arch Int Surg 2016; 6(2):84-9.

14. Kasaee A, Mirmohammadsadeghi A, Kazemnezhad F, Eshraghi B, Akbari M. R. The predictive factors of diplopia and extraocular movement limitations in isolated pure blow-out fracture. J Current Ophthalmology 2017; 29(1):54-8.

15. Tak KS, Jung MS, Lee BH, Kim JH, Ahn DK, Jeong HS, et al. Combination of Absorbable Mesh and Demineralized Bone Matrix in Orbital Wall Fracture for Preventing Herniation of Orbit. Craniofac Surgery 2014; 25(4):352-6.

16. Lee GHP, Ho SYM. Orbital Adherence Syndrome following the Use of Titanium Precontoured Orbital Mesh for the Reconstruction of Posttraumatic Orbital Floor Defects. Craniomaxillofac trauma reconstr 2017; 10(1):77-83.
17. He D, Li Z, Shi W, Sun Y, Zhu H, Lin M, et al. Orbitozygomatic fractures with enophthalmos: analysis of 64 cases treated late. J Oral Maxillofac Surgery 2012; 70(3):562-76.

18. Han X, Yang S, Dong Z, Feng W. Reconstruction of orbital blowout fractures using MEDPOR titanium mesh implants. Int J Clinical Exp Medicine 2016; 9(6): 12411-5.

19. Peng MY, Merbs SL, Grant MP, Mahoney NR. Orbital fracture repair outcomes with preformed titanium mesh implants and comparison to porous polyethylene coated titanium sheets. J Cranio-Maxillofac Surgery 2017; 45(2): 271-4.

20. Zavattero E, Ramieri G, Roccia F, Gerbino G. Comparison of the Outcomes of Complex Orbital Fracture Repair with and without a Surgical Navigation System: A Prospective Cohort Study with Historical Controls. Plast Reconstructive Surg 2017; 139(4):957-65.

Endereço para correspondência:

Lucas da Silva Barreto

Rua Clóvis Spínola, 40, bloco b, ap. 602

40080-240, Salvador, BA, Brasil

Telefone: (79) 99943-8317

E-mail: dr.Isbodonto@gmail.com

Recebido: 13/02/18. Aceito: 20/03/18. 